\title{
POTENCIAL EXPOSIÇÃO AO MERCÚRIO ATMOSFÉRICO NO AMBIENTE OCUPACIONAL DE COMÉRCIOS DE OURO DE PORTO VELHO, RONDÔNIA
}

\author{
Leidiane C. Lauthartte,,a, Diego F. Gomes ${ }^{\mathrm{a}}$, Marília H. Mussy ${ }^{\mathrm{a}}$, Ígor B. B. de Holandaa ${ }^{\mathrm{a}}$, Ronaldo Almeida ${ }^{\mathrm{b}}$ e Wanderley \\ R. Bastos ${ }^{\text {a }}$ \\ aDepartamento de Biologia, Universidade Federal de Rondônia, 76801-059 Porto Velho - RO, Brasil \\ bepartamento de Ciências Sociais e Ambientais, Universidade Federal de Rondônia, 76850-000 Guajará-Mirim - RO, Brasil
}

Recebido em 17/01/2018; aceito em 04/06/2018; publicado na web em 28/06/2018

\begin{abstract}
POTENTIAL EXPOSURE TO ATMOSPHERIC MERCURY IN THE OCCUPATIONAL ENVIRONMENT OF GOLD SHOPS IN PORTO VELHO, RONDÔNIA. The gold mining in the Madeira River basin is performed by dredges and ferries that extract from the bottom of the river alluvial gold which is amalgamated with mercury $(\mathrm{Hg})$. Throughout the process of gold purification, the $\mathrm{Hg}$ can be recovered by retort or crucible distillation. Subsequently, the gold is taken to the city of Porto Velho for commercialization, once more being smelted with blowtorch for jewelry making. This study aims to evaluate the Hg concentration exposed in the atmosphere of gold shops via an atomic absorption spectrophotometer coupled to portable cold steam generation (LUMEX) and to determine the occupational exposure through the analysis of urine samples of gold shops workers. Eleven gold shops and twenty workers were investigated. Some risky situations which may accompany the health of employees, such as poor ventilation, lack of exhaust systems and unhealthy places, have been verified in loco. The results showed atmospheric concentrations of $\mathrm{Hg}$ above the limits established by WHO, which is at most $200 \mathrm{ng} \mathrm{m}^{-3}$ in four gold shops. All examined presented total $\mathrm{Hg}$ results in the urine below the occupational limit of $\left(50.00 \mu \mathrm{g} \mathrm{L}^{-1}\right)$.
\end{abstract}

Keywords: mercury; gold commercialization; gold mining; Madeira River.

\section{INTRODUÇÃO}

Dentre os diversos metais pesados presentes no ambiente, o mercúrio $(\mathrm{Hg})$ é considerado pela Agência de Proteção Ambiental dos Estados Unidos (USEPA) um dos elementos químicos mais tóxicos, o qual apresenta efeitos prejudiciais à saúde humana, além de ser acumulativo e persistente no ambiente. ${ }^{1}$

$\mathrm{O} \mathrm{Hg}$ possui grande importância econômica devido ao uso industrial, principalmente nas indústrias farmacêuticas, eletroquímicas, praguicidas e indústrias de papel. ${ }^{2}$ Entretanto, é mais conhecido pela sua utilização em atividades de mineração de ouro, as quais expõem cerca de 15 milhões de trabalhadores de 70 países ao risco de intoxicação por Hg, colocando-o, na lista das Nações Unidas, entre as dez substâncias químicas que mais ameaçam a saúde do planeta. ${ }^{3}$

Desde agosto de 2017, entrou em vigor a Convenção de Minamata sobre $\mathrm{Hg}$, um tratado global assinado por 128 países para proteger a saúde humana e o ambiente dos efeitos adversos do $\mathrm{Hg}$. Através deste tratado, os países signatários, incluindo o Brasil, comprometeram-se em proibir novas minas de $\mathrm{Hg}$, eliminar progressivamente as minas já existentes, tomar medidas de controle sobre as emissões atmosféricas, e a regulamentar internacionalmente o setor informal para mineração artesanal e de ouro em pequena escala.

$\mathrm{O} \mathrm{Hg}$ pode ser emitido aos compartimentos naturais através do vapor resultante da queima do amálgama ouro-mercúrio (Au-Hg) nos garimpos e da fusão do ouro nos comércios de compra e venda das cidades em áreas de garimpo. As formas de contaminação humana pelo Hg são através do uso em ambiente ocupacional, pela inalação de vapores de $\mathrm{Hg}^{0}$ e pela contaminação alimentar, ${ }^{4,5}$ via ingestão do Hg orgânico pelos peixes e, posteriormente da ingestão dos peixes pela população. ${ }^{6-8}$

Ainda, $\mathrm{o} \mathrm{Hg}^{0}$ absorvido através da inalação de seus vapores é distribuído para o sangue, acumulando em altas concentrações no

*e-mail: leidianequimica@gmail.com cérebro e nos rins, ${ }^{2}$ podendo atravessar a barreira hemato-encefálica, causando danos ao sistema nervoso central. ${ }^{9} \mathrm{O}$ grau de contaminação por essa via de exposição pode ser determinado por meio de dosimetria da urina ou do sangue. ${ }^{10,11,8}$

Inserida nesse contexto, a bacia do Alto rio Madeira em Rondônia foi a segunda maior região produtora de ouro de garimpo na Amazônia, ${ }^{12}$ perdendo apenas para a bacia do rio Tapajós no Pará. A exploração iniciou-se em meados da década de 1970 e provocou uma das maiores ondas de migração da região Amazônica devido à descoberta de grandes reservas de ouro aluvionar na calha do rio Madeira. ${ }^{13}$

A exploração de ouro atingiu seu máximo de produção na década de 1980 com 100 toneladas por ano. ${ }^{14,15}$ Entre as décadas de 1980 e 1990, a produção foi diminuindo devido à queda no preço e esgotamento das reservas de ouro, chegando a 30 toneladas/ano ao final dos anos 90. . $^{16,14}$

Em contrapartida, a emissão de $\mathrm{Hg}$ para o meio ambiente na década de 80 , no rio Madeira, chegava a 130 toneladas/ano. ${ }^{17,18}$ Estima-se que somente entre os anos 80 e início dos 90 a quantidade de $\mathrm{Hg}$ emitida no ambiente tenha ultrapassado as 2.000 toneladas. ${ }^{19,14}$

Estudos pretéritos evidenciaram que o fator de emissão médio de $\mathrm{Hg}$ calculado na atividade de garimpo de ouro na década de 80 foi de, aproximadamente, 1,3 a 2,0 $\mathrm{kg}$ de $\mathrm{Hg}^{0}$ para $1,0 \mathrm{~kg}$ de ouro produzido. ${ }^{17,18,20}$ Neste contexto, aproximadamente, 55 a $65 \%$ do $\mathrm{Hg}^{0}$ era emitido para a atmosfera durante a fusão do amálgama de $\mathrm{Au}-\mathrm{Hg}$ e 35 a $45 \%$ eram lançados diretamente para os rios, solos e drenagens da região. ${ }^{19}$

Atualmente, o $\mathrm{Hg}$ utilizado no processo de garimpo de ouro é reutilizado após a destilação em retortas (destiladores ou cadinhos), que quando mal manuseadas, podem desprender vapor de $\mathrm{Hg}^{0}$ para a atmosfera, além de causar a exposição do operador. $\mathrm{O}$ mesmo acontece em escala menor com a fusão do ouro nos comércios especializados.

O processo de extração de ouro aluvionar no rio Madeira é realizado através de dragas com desagregadores rotativos com alcance 
de 30 a $40 \mathrm{~m}$ de profundidade. A dragagem ocorre por cerca de 20 a 24 horas e o material obtido é separado gravimetricamente em calhas de madeira inclinadas forradas com carpetes onde o sedimento mais pesado contendo ouro ficará retido.

Após a separação gravimétrica, é feito o batimento dos carpetes e o material extraído é colocado em tambores. A apuração do ouro é realizada através do método de amalgamação com $\mathrm{Hg}$ em tambor com o uso de um agitador mecânico. Após a amalgamação, é feita a separação do amálgama e a filtração (expremeção) do $\mathrm{Hg}$ em excesso com um pano de algodão. O amálgama $\mathrm{Au}-\mathrm{Hg}$ (contendo entre 30 a $50 \%$ de $\mathrm{Hg}$ em peso) é então submetido à sublimação em retorta aquecida pelo gás butano e com recuperação do $\mathrm{Hg}$ em condensador simples (borbulhamento do gás contendo $\mathrm{Hg}$ em balde de água fria). ${ }^{21}$

A mineração de ouro no rio Madeira ocorre em uma escala inferior ao registrado nos anos entre 1980 e $1990,{ }^{22}$ porém, os problemas com o Hg relacionados à saúde do trabalhador e aos impactos causados ao meio ambiente ainda preocupam a comunidade científica, devido à sua toxicidade e também à falta de fiscalização pelos órgãos responsáveis.

Mediante o histórico de garimpagem de ouro na região de Porto Velho (RO), existem diversos comércios varejistas especializados, que compram o ouro diretamente de garimpeiros, que realizam a purificação do ouro em seu comércio, o que pode expor os trabalhadores a elevadas concentrações de vapor de $\mathrm{Hg}$. No intuito de investigar se ocorre a exposição ocupacional ao $\mathrm{Hg}$, o objetivo deste estudo foi avaliar as concentrações de $\mathrm{Hg}$ na atmosfera e na urina dos profissionais de comércios de ouro.

\section{PARTE EXPERIMENTAL}

\section{Área de estudo}

A área de estudo está localizada no município de Porto Velho, Estado de Rondônia (Figura 1) que está sob influência do clima do tipo Aw - clima tropical chuvoso, com média anual da precipitação pluvial variando entre 1.400 e $2.500 \mathrm{~mm} / \mathrm{ano}$, e a média anual da temperatura do ar entre 24 e $26^{\circ} \mathrm{C}$, sendo que as maiores precipitações ocorrem nos meses de dezembro a março e o período de estiagem se estende de julho a setembro. ${ }^{23}$

A população de Porto Velho é de 428.527 mil habitantes. ${ }^{24}$ Porto Velho é a capital de Rondônia e é a terceira economia da Região Norte e registrou um crescimento de $30,2 \%$ no Produto Interno Bruto (PIB), atingindo R \$ 6,6 bilhões. ${ }^{24} \mathrm{~A}$ atividade econômica predominante no município é a do setor de serviços, concentrando $83,8 \%$ das atividades. Deve-se ressaltar, porém, que a economia local conta ainda com a indústria extrativa do minério (como a de cassiterita e de ouro); a pesca e a agricultura (produção agrícola: arroz, mandioca, milho e abacaxi).

O número de comércios especializados na compra e venda de ouro reduziu bastante após o declínio do ciclo de ouro no município. São cerca de 30 lojas comercializadoras que estão localizadas, uma ao lado da outra, no centro da capital, nas avenidas Campos Sales (Longitude: 400852,13 e Latitude: 9031039,82) e Joaquim Nabuco (Longitude: 401201,61 e Latitude: 9031357,22). A distribuição espacial dos pontos de coleta em comércios de ouro é apresentada na Figura 2.

\section{Métodos}

As concentrações atmosféricas de $\mathrm{Hg}$ nos comércios de ouro foram obtidas utilizando-se o analisador Zeeman portátil de mercúrio, modelo Lumex RA-915M (Lumex Instruments, Saint Petersburg, Rússia), que é um analisador de mercúrio gasoso $\left(\mathrm{Hg}^{0}\right)$ no ar baseado na técnica de espectrometria de absorção atômica com efeito Zeeman (ZAAS-HFM), utilizando uma modulação de alta frequência de polarização da luz. Este equipamento possui limites de detecção muito baixos de $0,5 \mathrm{ng} \mathrm{m}^{-3} \mathrm{e}$ opera em tempo real para gases e ar atmosférico, não necessecitando de pré-tratamento ou concentração das amostras. ${ }^{25}$

Desta forma, foram avaliados diferentes pontos dos comércios formando-se um conjunto de avaliação desde a entrada da loja (calçada), recepção (entrada) e a sala de queima do ouro ou polimento

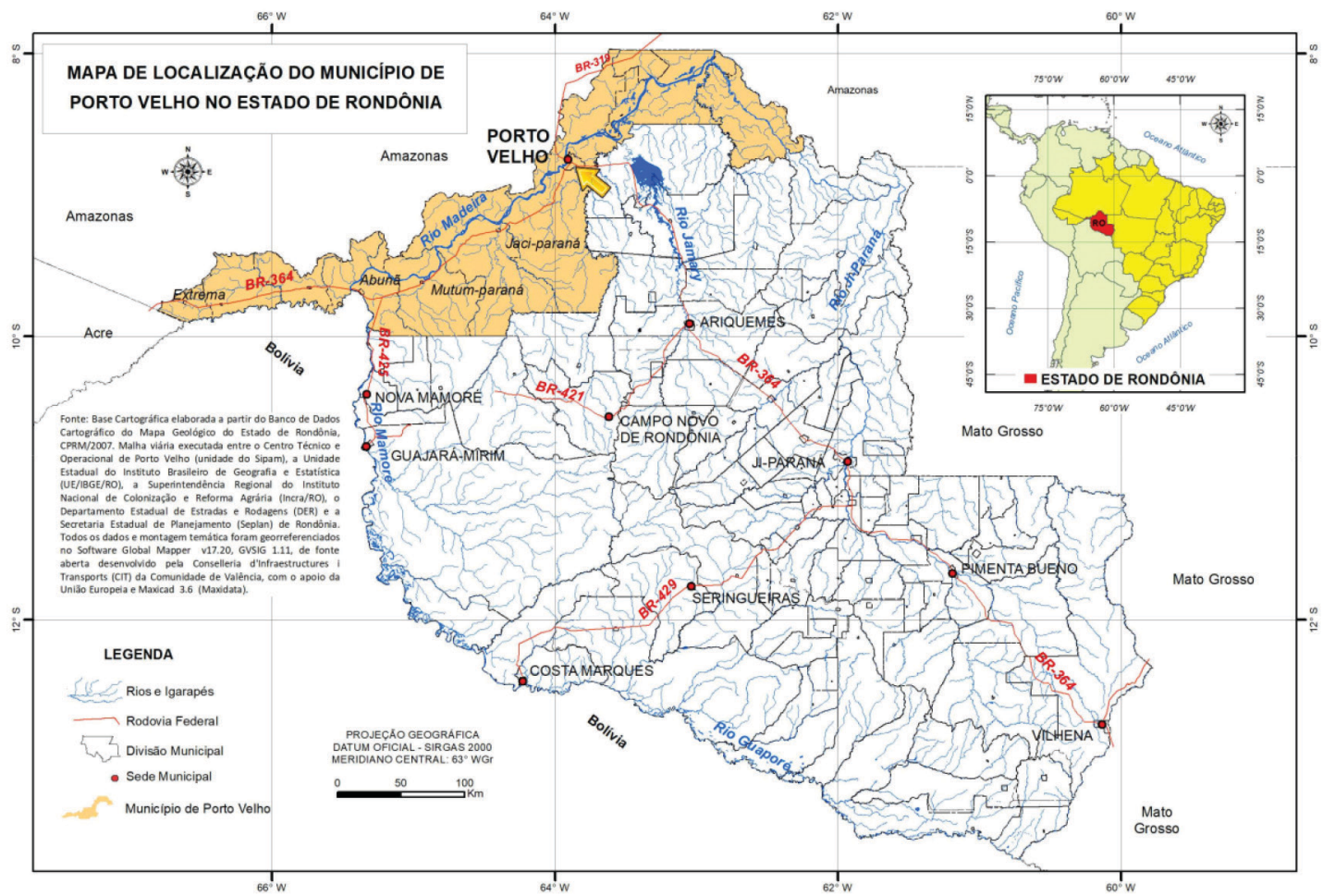

Figura 1. Mapa de localização do município de Porto Velho no estado de Rondônia. Fonte: Secretaria Estadual de Planejamento (Seplan) de Rondônia. Google Earth, acesso em 18/09/2017 


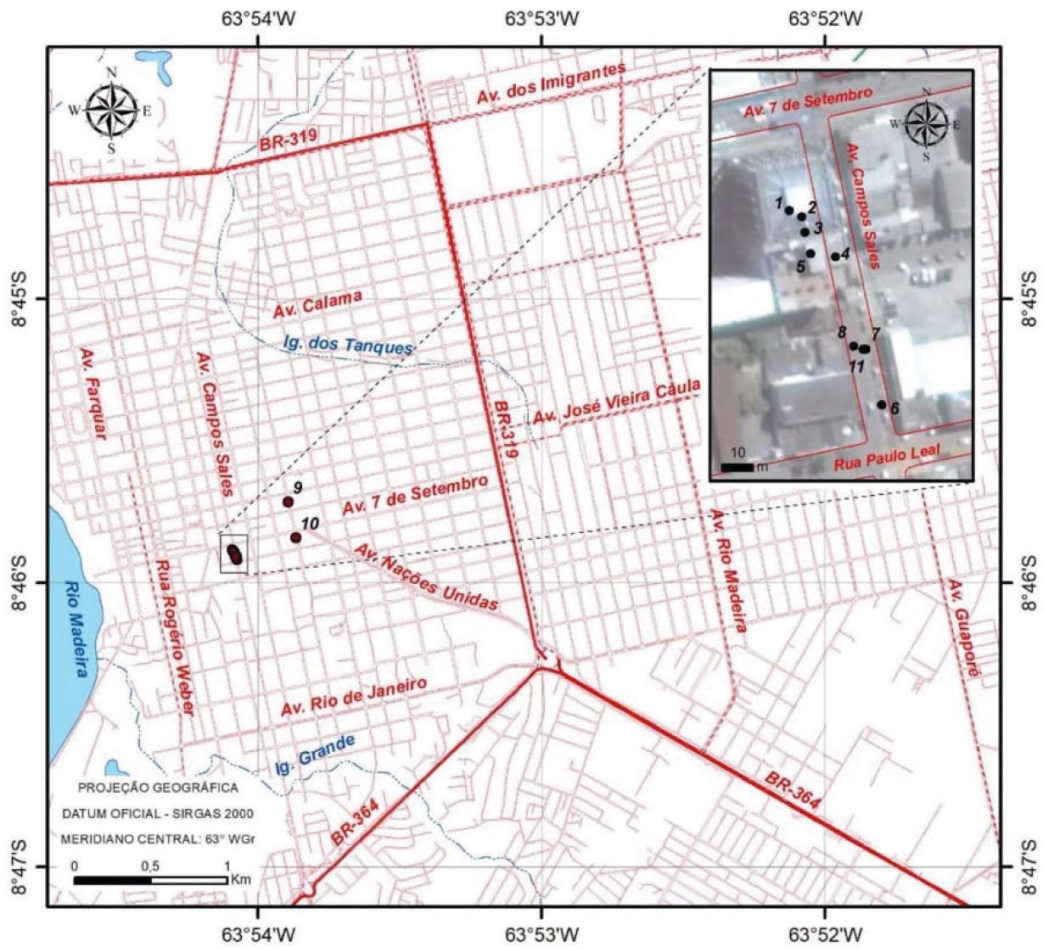

Figura 2. Mapa da localização dos comércios de ouro estudados no município de Porto Velho/RO. Fonte: Secretaria Estadual de Planejamento (Seplan) de Rondônia. Google Earth, acesso em 18/09/2017

de joias (polimento). A coleta foi realizada pela manhã no período de seca na região (out/2015 e nov/2016), em dias ensolarados, com temperaturas acima de $30^{\circ} \mathrm{C}$. Padronizaram-se as medições de $\mathrm{Hg}^{0}$ atmosférico a uma altura de $50 \mathrm{~cm}$ do chão. As integrações ocorreram a cada 10 segundos durante 3 minutos em cada ponto amostral. As medições em tempo real foram feitas com a visualização do processo em um display digital do aparelho e a gravação dos dados foi feita por conexão do instrumento ao laptop.

Este estudo foi realizado em 11 comércios de compra e venda de ouro da cidade de Porto Velho.

Para avaliar a exposição ocupacional aos vapores de $\mathrm{Hg}^{0}$ também foram coletadas e analisadas amostras de urina de 20 trabalhadores. Para isso, esta pesquisa foi submetida ao Comitê de Ética em Pesquisa da Fundação Universidade Federal de Rondônia (Parecer CAAE 48095315.3.0000.5300) e aprovada em 14/12/2015. Todos os sujeitos participantes desta pesquisa receberam orientações de acordo com a Resolução n 466, de 12 de dezembro de 2012 e assinaram o Termo de Consentimento Livre e Esclarecido (TCLE). Os resultados obtidos nesta pesquisa foram entregues a cada participante individualmente e sigilosamente.

Na Tabela 1 são apresentadas algumas informações e características relevantes sobre os comércios de ouro avaliados em Porto Velho.

Durante a purificação do ouro nas lojas de compra, a utilização de maçarico (mistura de gases acetileno e oxigênio) provoca a volatilização do $\mathrm{Hg}$ residual (2 a 7\%). ${ }^{26} \mathrm{~A}$ inexistência de capelas e exaustores adequados nesses estabelecimentos provoca a contaminação dos ambientes de trabalho nas áreas urbanas.

As amostras de urina foram coletadas pelos próprios trabalhadores e armazenadas em frascos de polipropileno de $40 \mathrm{~mL}$, de acordo com a metodologia recomendada. ${ }^{27}$ As amostras foram mantidas resfriadas

Tabela 1. Características dos comércios de ouro estudados de Porto Velho. Sistema de Coordenadas Planas, projeção Universal Transverso de Mercator (UTM) Datum SAD 69, Zona 20 L

\begin{tabular}{|c|c|c|c|c|c|}
\hline \multirow{2}{*}{ Comércio } & \multicolumn{2}{|c|}{ Coordenadas Geográficas } & \multirow{2}{*}{$\mathrm{N}^{\mathrm{o}}$ de Colaboradores } & \multirow{2}{*}{$\begin{array}{l}\text { Há quanto tempo existe? } \\
\qquad(\text { anos) }\end{array}$} & \multirow{2}{*}{$\begin{array}{l}\text { Possui capela de exaustão/ } \\
\text { sistema de exaustão? }\end{array}$} \\
\hline & Longitude & Latitude & & & \\
\hline 1 & 400852,13 & 9031039,82 & 03 & 15 & Não \\
\hline 2 & 400851,48 & 9031038,16 & 03 & 20 & Sim \\
\hline 3 & 400846,54 & 9031030,41 & 01 & 30 & Não \\
\hline 4 & 400851,28 & 9031027,55 & 01 & 10 & Não \\
\hline 5 & 400854,03 & 9031028,77 & 01 & 14 & Não \\
\hline 6 & 400870,14 & 9031006,58 & 03 & 20 & Não \\
\hline 7 & 400875,77 & 9030999,41 & 01 & 01 & Não \\
\hline 8 & 400867,84 & 9031001,71 & 01 & 26 & Sim \\
\hline 9 & 401201,61 & 9031357,22 & 05 & 30 & Sim \\
\hline 10 & 401258,38 & 9031118,74 & 03 & 20 & Não \\
\hline 11 & 400865,88 & 9030994,41 & 02 & 08 & Não \\
\hline
\end{tabular}


em caixa térmica e depois congeladas a $-4{ }^{\circ} \mathrm{C}$ até o momento da determinação no prazo máximo de 30 dias.

Para determinação das concentrações de $\mathrm{Hg}$ total $(\mathrm{Hg}-\mathrm{T})$ na urina, utilizou-se 2,0 $\mathrm{mL}$ da amostra com adição de $4,0 \mathrm{~mL}$ de solução $\mathrm{HNO}_{3}: \mathrm{H}_{2} \mathrm{SO}_{4}$ (1:1, Merck) em bloco digestor (Tecnal, Mod. 007A, São Paulo, Brazil) por $30 \mathrm{~min}$ a $60{ }^{\circ} \mathrm{C}$ e $5,0 \mathrm{~mL}$ de $\mathrm{KMnO}_{4}$ a $5 \%\left(\mathrm{~m} / \mathrm{v}\right.$, Merck) no bloco digestor por mais $20 \mathrm{~min}$ a $60{ }^{\circ} \mathrm{C} .{ }^{28}$ Após este processo, as amostras foram deixadas em descanso (overnight) e acrescentadas algumas gotas da solução de $\mathrm{NH}_{2} \mathrm{OH}$. $\mathrm{HCl} 12 \%$ (m/v, Merck). A determinação de $\mathrm{Hg}$ total foi realizada em espectrofotômetro de absorção atômica com geração de vapor frio com sistema de injeção em fluxo (FIAS) (CV-AAS, Flow Injection Mercury System FIMS-400 Perkin-Elmer, Germany), e amostrador automático (AS90) utilizando Software (Winlab-Perkin Elmer).

Para assegurar a qualidade das análises químicas de urina, o material utilizado foi descontaminado com $\mathrm{HNO}_{3} 10 \%$ (m/v, Merck), e enxaguados com água ultrapura. As amostras foram realizadas em duplicatas e utilizados brancos para controle analítico.

Para o controle analítico de exatidão das análises de $\mathrm{Hg}$ total nas urinas, utilizou-se as amostras de referências de cabelo e de peixes certificadas pela Agência Internacional de Energia Atômica (IAEA 086), com valor de referência de $0,57 \mathrm{mg} \mathrm{kg}^{-1}$ e Tuna Fish (BCR-463) com valor de referência de $2,85 \mathrm{mg} \mathrm{kg}^{-1}$. A recuperação analítica média dessas amostras certificadas durante o processo de análise para $\mathrm{Hg}$-T foi de $0,53 \pm 0,02 \mathrm{mg} \mathrm{kg}^{-1}$ (Recuperação média de $93 \%$ ) para a IAEA O86 e 2,72 $\pm 0,02 \mathrm{mg} \mathrm{kg}^{-1}$ (Recuperação média de 97 $\%)$ para a BCR-463. O LDT (limite de detecção da técnica) foi de $0,002 \mathrm{mg} \mathrm{kg}^{-1}$. As análises de urina foram realizadas no Laboratório de Biogeoquímica Ambiental Wolfgang C. Pfeiffer, que participa do Programa Interlaboratorial de Controle de Qualidade Analítica da Espanha (PICC-MetU) realizando análises de amostras de urina para Hg periodicamente, desde 2001, com ótimo desempenho.

Para tratamento dos dados foram utilizados os programas Graph Pad Prism 5.0 e Excel 2007. Foram realizados testes para verificação da normalidade ou não normalidade dos dados (Shapiro-Wilk). Como a maioria dos dados não segue uma distribuição normal, o teste não paramétrico de Kruskal-Wallis foi aplicado para verificar diferenças entre os grupos.

\section{RESULTADOS E DISCUSSÃO}

A Figura 3 apresenta os dados das concentrações de $\mathrm{Hg}$ referente à entrada das lojas (calçada). O primeiro ponto amostral em cada comércio foi na calçada, do lado de fora, em frente ao comércio. Concentrações acima de $200 \mathrm{ng} \mathrm{m}^{-3} \mathrm{de} \mathrm{Hg}$ foram observadas nas lojas 8, 10 e 11 . Ocorreram diferenças significativas entre concentrações médias de $\mathrm{Hg}^{0}$ nas calçadas das 11 lojas comercializadoras de ouro estudadas $(\mathrm{p}<0,0001)$ com destaque para as lojas 4, 6, 7, 8, 10 e 11 que apresentaram concentrações médias de $\mathrm{Hg}^{0}$ mais elevadas em relação às demais lojas (Figura 3).

A Figura 4 mostra os dados das concentrações de $\mathrm{Hg}$ referente ao segundo ponto amostral, na recepção das lojas (entrada). Concentrações acima de $200 \mathrm{ng} \mathrm{m}^{-3} \mathrm{de} \mathrm{Hg}$ foram observadas nas lojas 8,9 e 10. Ocorreram diferenças significativas na entrada das 11 lojas de comércios de ouro ( $\mathrm{p}<0,0001)$ com destaque para as lojas 3,6 , 8, 9, 10 e 11 que apresentaram concentrações médias de $\mathrm{Hg}^{0}$ mais elevadas em relação às demais lojas (Figura 4).

A Figura 5 mostra os dados das concentrações de $\mathrm{Hg}$ referente às salas de polimento, onde ocorrem a fusão do ouro e o polimento de joias. Ocorreram diferenças significativas entre as 11 salas de polimento das lojas avaliadas $(\mathrm{p}<0,0001)$ com destaque para as lojas $6,8,9,10$ e 11 que apresentaram concentrações médias de $\mathrm{Hg}^{0}$ mais elevadas em relação às demais lojas (Figura 4).
Hg-T (calçada)

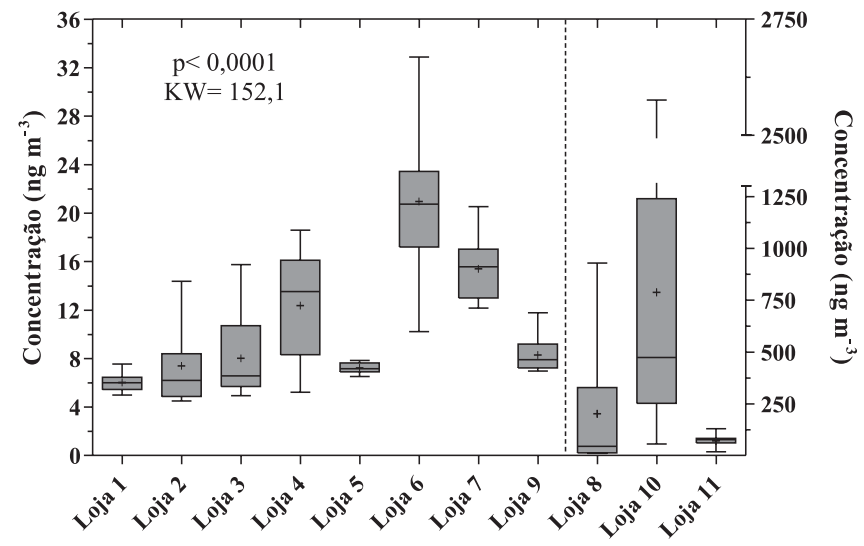

Figura 3. Box plot representando as concentrações de $\mathrm{Hg}^{0}\left(\mathrm{ng} \mathrm{m}^{-3}\right)$ no ar atmosférico na calçada dos comércios de compra e venda de ouro de Porto Velho, Rondônia. O eixo Y secundário (direita) corresponde às concentrações das lojas 8, 10 e 11, enquanto que o eixo Y principal (esquerda) refere-se às concentrações das demais lojas avaliadas

\section{Hg-T (entrada)}

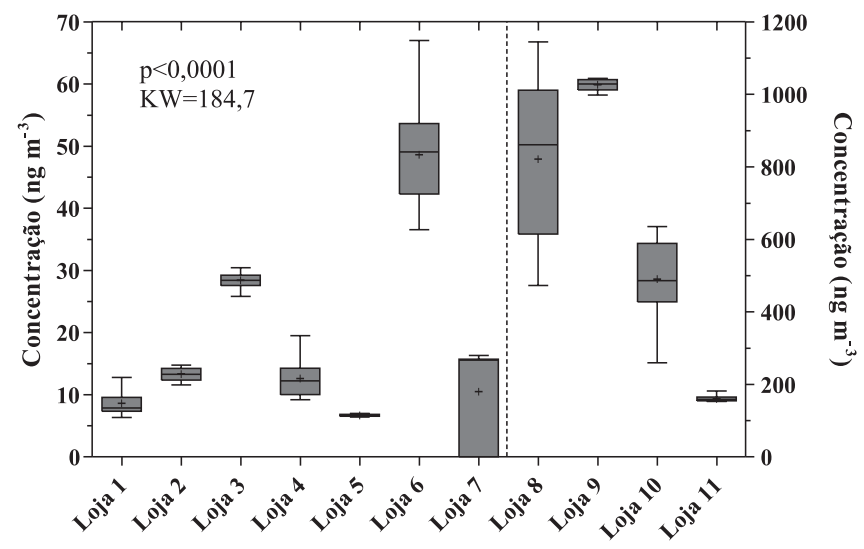

Figura 4. Box plot representando às concentrações de $\mathrm{Hg}^{0}$ (ng $\left.\mathrm{m}^{-3}\right)$ no ar atmosférico próximo a entrada dos comércios de compra e venda de ouro de Porto Velho, Rondônia. O eixo Y secundário (direita) corresponde às concentrações das lojas 8, 9, 10 e 11, enquanto que o eixo Y principal (esquerda) refere-se às concentrações das demais lojas avaliadas

Hg-T (polimento)

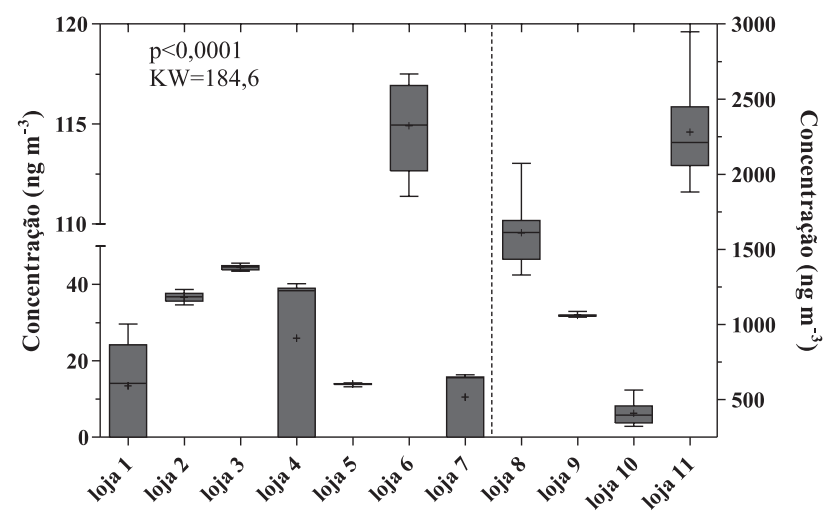

Figura 5. Box plot representando às concentrações de $\mathrm{Hg}^{0}\left(\mathrm{ng} \mathrm{m}^{-3}\right)$ no ar atmosférico na sala de polimento dos comércios de compra e venda de ouro de Porto Velho, Rondônia. O eixo Y secundário (direita) corresponde às concentrações das lojas 8, 9, 10 e 11, enquanto que o eixo Y principal (esquerda) refere-se às concentrações das demais lojas avaliadas 
Uma avaliação no entorno dos comércios estudados demonstrou que as concentrações de $\mathrm{Hg}$ variaram de 5,41 a 7,20 ng m ${ }^{-3}$, evidenciando que a contaminação por $\mathrm{Hg}$ é pontual, somente no interior de alguns comércios.

Segundo Lacerda e Marins ${ }^{29}$ as emissões de $\mathrm{Hg}$ de mineração de ouro são praticamente restritas à região amazônica, que resulta em uma alta emissão por área de aproximadamente $16 \mathrm{~g} \mathrm{~km}^{-2}$, e taxas de deposição atmosférica maiores do que em áreas industriais.

$\mathrm{O}$ estudo de Artaxo et al. mostrou que as concentrações de $\mathrm{Hg}$ de áreas virgens e áreas que não estejam localizadas próximas às regiões de mineração de ouro apresenta valores de background de $\mathrm{Hg}$ variando de 0,5 a 2,0 $\mathrm{ng} \mathrm{m}^{-3} \mathrm{e}$, nas áreas que são fortemente impactadas pela atividade de mineração de ouro ou de queima de biomassa as concentrações de $\mathrm{Hg}$ total variaram de 5,0 a $14,0 \mathrm{ng} \mathrm{m}^{-3} .{ }^{30}$

Considerando os três pontos amostrais em cada comércio, o comércio 5 apresentou os menores valores médios de $\mathrm{Hg}$ atmosférico, $6,86 \mathrm{ng} \mathrm{m}^{-3}$ e o comércio 8 apresentou os maiores valores médios, $878,85 \mathrm{ng} \mathrm{m}^{-3} \mathrm{de} \mathrm{Hg}$. A concentração mais alta foi no comércio 10, com 2.576,24 ng m${ }^{-3}$ de $\mathrm{Hg}$, na sala de polimento.

No entanto, esses resultados são inferiores aos obtidos na pesquisa de Malm (1991), ${ }^{19}$ na qual foram encontradas concentrações de $\mathrm{Hg}$ variando de 450 a $7.500 \mathrm{ng} \mathrm{m}^{-3}$, com média de $2.800 \mathrm{ng} \mathrm{m}^{-3}$, no ar urbano próximo aos comércios de ouro na cidade de Porto Velho/RO e concentrações de $\mathrm{Hg}$ entre 100.000 a $107.200 \mathrm{ng} \mathrm{m}^{-3}$, no ambiente ocupacional nos comércios durante a fusão do ouro. Esses resultados indicam que, atualmente, a exposição ocupacional ao $\mathrm{Hg}$ é inferior ao auge da atividade garimpeira de ouro no rio Madeira, fato que se deve à redução no volume de ouro comercializado na região, melhoramento nos processos de amalgamação e recuperação do $\mathrm{Hg}$ utilizado através dos cadinhos (retorta), bem como às legislações impostas pelo Estado e seus órgãos ambientais fiscalizadores.

A Organização Mundial de Saúde (OMS) define uma concentração tolerável de $200 \mathrm{ng} \mathrm{m}^{-3}$ para exposição por inalação em longo prazo para vapor de $\mathrm{Hg}$ e a legislação brasileira NR-15 dispõe sobre o limite de tolerância no local de trabalho $(\mathrm{Hg}$, todas as formas exceto orgânicas) de $0,04 \mathrm{mg} \mathrm{m}^{-3}$ (40.000 $\mathrm{ng} \mathrm{m}^{-3}$ ) até 48 horas/semana. ${ }^{31,32}$

Ainda que as concentrações de $\mathrm{Hg}$ atmosférico tenham diminuído em relação a década de 80 e 90 , as concentrações de vapor de $\mathrm{Hg}$ em certos ambientes de comercialização de ouro em Porto Velho continuam altas, acima do recomendado pela OMS, o que pode em longo prazo provocar danos à saúde dos trabalhadores.

A Tabela 2 mostra a estatística descritiva da concentração de Hg-T nas amostras de urina dos 20 trabalhadores das lojas de comercialização de ouro de Porto Velho.

Tabela 2. Estatística descritiva para as concentrações de $\mathrm{Hg}-\mathrm{T}\left(\mu \mathrm{g} \mathrm{L}^{-1}\right)$ das amostras de urina $(\mathrm{n}=20)$

\begin{tabular}{cc}
\hline Mínimo & 0,46 \\
25\% Percentil & 1,06 \\
Mediana & 2,15 \\
$75 \%$ Percentil & 5,06 \\
Máximo & 15,12 \\
Média & 4,10 \\
\hline
\end{tabular}

Os limites biológicos de tolerância preconizados pela OMS são relacionados às concentrações de $\mathrm{Hg}$ na urina; limite normal é até $10,00 \mu \mathrm{g} \mathrm{L} \mathrm{L}^{-1}$ e o limite ocupacional até $50,00 \mu \mathrm{g} \mathrm{L}^{-1} .{ }^{31}$ Apesar da exposição aos vapores de $\mathrm{Hg}$ no ambiente de trabalho, os profissionais apresentaram indíces de $\mathrm{Hg}$ na urina abaixo do limite ocupacional segundo a OMS. O teste $\mathrm{t}$ realizado revelou que as concentrações de $\mathrm{Hg}$ total na urina não diferem significativamente entre os comércios investigados ( $\mathrm{p}=0,0562$, Mann-Whitney $\mathrm{U}=129,0$ e coeficiente de determinação, $r^{2}=0,1318$ ) (Figura 6).

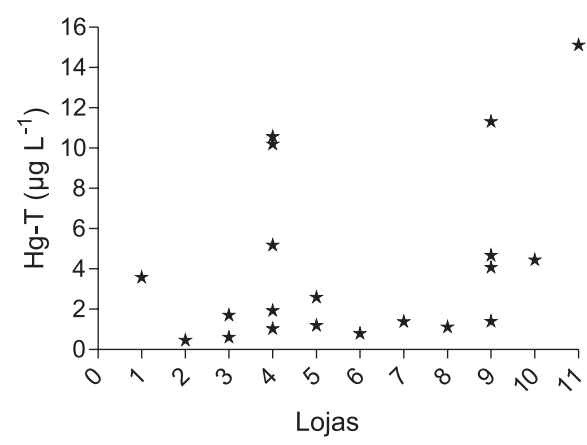

Figura 6. Correlação entre as concentrações de Hg-T na urina dos profissionais dos comércios investigados

Durante a realização desta pesquisa, foi observado que nenhum dos ouríves realizava as atividades fazendo uso de equipamentos de proteção individual. Apenas em três comércios existiam sistemas de exaustão e as condições de trabalho em algumas lojas eram bastante precárias, como pode ser observado na Figura 7.
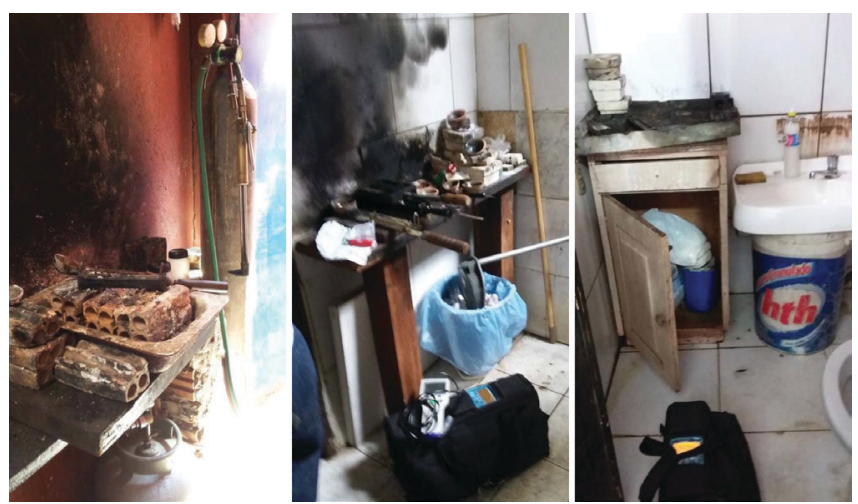

Figura 7. Condições onde ocorre a queima do ouro nos comércios de ouro. Fonte: Leidiane Caroline Lauthartte

Segundo relatos dos sujeitos desta pesquisa, não há nenhum tipo de fiscalização ou monitoramento das atividades realizadas nesses comércios, nem a avaliação de $\mathrm{Hg}$ atmosférico. Estas informações foram confirmadas junto aos órgãos de Vigilância Sanitária Municipal (SEMUSA) e Estadual (AGEVISA), bem como no CEREST Estadual de Rondônia (Centro de Referência em Saúde do Trabalhador de Rondônia). Não há dados pretéritos divulgados por estes órgãos e, no momento, não há ações realizadas ou a serem implantadas por esses órgãos quanto ao uso ou monitoramento atmosférico do $\mathrm{Hg}$.

Contudo, medidas preventivas como a utilização de equipamentos de proteção individual e coletivos, ambientes de trabalho ventilados e refrigerados, devem ser tomadas visando à redução da exposição aos vapores de Hg. Desta forma, faz-se necessário o contínuo monitoramento dos ambientes ocupacionais como prevenção da saúde do trabalhador.

\section{CONCLUSÃO}

As concentrações de $\mathrm{Hg}$ atmosférico no interior dos comércios de ouro variaram de 6,86 a 2.576,24 $\mathrm{ng} \mathrm{m}^{-3}$ e valores acima de 200,00 ng $\mathrm{m}^{-3}$ foram observados em 4 comércios, do total de 11 investigados. Os trabalhadores avaliados nesta pesquisa apresentaram concentrações de $\mathrm{Hg}$ total na urina abaixo do limite ocupacional até $50,00 \mu \mathrm{g} \mathrm{L}^{-1}$ fixado pela OMS. 
Verificou-se, com essa pesquisa, a necessidade de fiscalização, pelos órgãos de vigilância à saúde e monitoramento, dos níveis de $\mathrm{Hg}$ nos ambientes ocupacionais, com vistas à promoção da saúde pública.

\section{AGRADECIMENTOS}

Os autores agradecem ao CNPQ (Conselho Nacional de Desenvolvimento Científico e Tecnológico), proc. (458977/2014-4) pelo apoio financeiro concedido, à Coogarima (Cooperativa dos Garimpeiros do Rio Madeira), a Charles da Silva Barata pela confecção dos mapas e a Rafael Marques Almeida pela revisão.

\section{REFERÊNCIAS}

1. https://www.epa.gov/sites/production/files/2015-09/documents/volume7. pdf, acessada em junho 2018.

2. Cooperação Técnica entre Brasil, Bolívia e Colômbia: Teoria e Prática para o Fortalecimento da Vigilância em Saúde de Populações Expostas a Mercúrio, Organização Pan-Americana da Saúde: Brasília, 2011.

3. https://nacoesunidas.org/entra-em-vigor-convencao-global-sobrereducao-do-uso-de-mercurio/, acessada em junho 2018.

4. Hacon, S.; Artaxo, P.; Gerald, F.; Yamasoe, M. A.; Campos, R. C.; Conti, L. F.; Lacerda, L. D.; Water, Air, Soil Pollut. 1995, 80, 273.

5. Gochfeld, M.; Ecotoxicol. Environ. Saf. 2003, 56, 174.

6. Oliveira, R. C.; Dórea, J. G.; Bernardi, J. V. E.; Bastos, W. R.; Almeida, R.; Manzatto, Â. G.; Ann. Hum. Biol. 2010, 37, 629.

7. Vieira, S. M.; Almeida, R.; Holanda, I. B. B.; Mussy, M. H.; Galvão, R. C. F.; Crispim, P. T. B.; Dorea, J. G.; Bastos, W. R.; Int. J. Hyg. Environ. Health 2013, 216, 682.

8. Li, P.; Yang, Y.; Xiong, W.; Biol. Trace Elem. Res. 2015, 168, 330.

9. Aschner, M.; Aschner, J. L.; Neurosci. Biobehav. Rev. 1990, 14, 2, 169.

10. Gonçalves, A.; Gonçalves, N. N. da S.; Revista Panamericana de Salud Pública 2004, 16, 6, 415.

11. Shirkhanloo, H.; Golbabaei, F.; Hassani, H.; Eftekhar, F.; Kian, M. J.; Iran J. Public Health 2014, 43, 793.

12. Bastos, W. R.; Lacerda, L. D.; Geochim. Bras. 2004, 18, 99.
13. Lacerda, L. D.; Bastos, W. R. Em Bioremediation of Mercury Current Research and Industrial Applications; Wagner-Döbler., I., eds.; Caister Academic Press: Poole, 2013, cap. 4.

14. Wasserman, J. C.; Hacon, S. S.; Wasserman, M. A.; Mundo \& Vida 2001, $2,46$.

15. Lacerda, L. D.; Env. Geol. 2003, 43, 308.

16. Veiga, M. M.; Introducing New Technologies for Abatement of Global Mercury Pollution in Latin America, UNIDO/UBC/CETEM/CNPq: Rio de Janeiro, 1997.

17. Pfeiffer, W. C; Lacerda, L. D.; Environ. Technol. Lett. 1988, 9, 325.

18. Pfeiffer, W. C; Lacerda, L. D; Malm, O.; Souza, C. M. M.; Silveira, E. G. Bastos, W. R.; Sci. Total Environ. 1989, 87/88, 233.

19. Malm, O.; Tese de Doutorado, Universidade Federal do Rio de Janeiro, Brasil, 1991.

20. Lacerda, L. D.; Pfeiffer, W. C.; Quim. Nova 1992, 15, 155.

21. Aspectos diversos da garimpagem de ouro. CETEM/CNPq: Rio de Janeiro, 1992.

22. Wanderley, L. J. M.; Tese de Doutorado, Universidade Federal do Rio de Janeiro, Brasil, 2015.

23. Atlas Geoambiental de Rondônia, SEDAM: Porto Velho, 2002.

24. Censo demográfico 2010, IBGE: Rio de Janeiro, 2010.

25. Sholupov, S.; Pogarev, S.; Ryzhov, V.; Mashyanov, N.; Stroganov, A.; Fuel Process. Technol. 2004, 8, 473.

26. Lacerda, L. D.; Quim. Nova 1997, 20, 196.

27. Campos, R. C.; Pivetta, F. Em Mercúrio em Áreas de Garimpos de Ouro, Série Vigilância, 12; Câmara, V. M., ed.; Metapec, Centro Panamericano de Ecologia Humana e Saúde: Brasília, 1993, cap. 6.

28. Bastos, W. R.; Malm, O.; Pfeiffer, W. C.; Cleary, D.; Ciência e Cultura 1998, 50, 4, 255.

29. Lacerda, L. D.; Marins, R. V.; J. Geochem. Explor. 1997, 58, 223.

30. Artaxo, P.; Campos, R. C.; Fernandes, E. T.; Martins, J. V.; Xiao, Z.; Lindqvist, O.; Fernández-Jiménez, M. T.; Maenhaut, W.; Atmos. Environ. 2000, 34, 4035.

31. http://www.who.int/ipcs/publications/cicad/en/cicad50.pdf, acessada em junho 2018.

32. http://www.saude.mt.gov.br/arquivo/1875, acessada em junho 2018. 